Hinsichtlich der Bewegungen von $6 I_{1}$ und $6 I_{2}$ Cygni sind aus meinen Messungen die folgenden Resultate zu ziehen. Betreffs der relativen Bewegung von $6 I_{2}$ inbezug auf $6 I_{1}$ ergibt sich:

$$
\begin{aligned}
& \mu=+0.0034 \pm 0.0047 \\
& \mu^{\prime}=-0.1510 \pm 0.0057 .
\end{aligned}
$$

Herr Dunér hatte bekanntlich eine Krümmung der relativen Bewegung nachgewiesen ${ }^{1}$ ), die durch die Untersuchungen des Herrn O. Struve bestätigt ward. C. F. W. Peters hat dke Struveschen Beobachtungen durch eine elliptische Bahn darzustellen versucht ${ }^{2}$ ), und zwar gibt die Peterssche Ephemeride für 1902.0 etwa:

$$
\begin{aligned}
& \mu=+0.008 \\
& \mu^{\prime}=-0.169 .
\end{aligned}
$$

Die Veränderlichkeit der relativen Bewegung, die von seiten einiger Astronomen bestritten worden ist, wird also durch meine Messungen im Verein mit den früheren bestätigt.

Für die absolute Eigenbewegung von 6 $\mathbf{I}_{1}$ Cygni hatte ich bei der Reduktion meiner Beobachtungen nach dem Berliner Jahrbuch die Werte

$$
\begin{aligned}
& \mu_{\alpha}=+0^{\mathrm{s}} 3443 \\
& \mu_{\delta}=+3^{\prime \prime} 240^{\circ}
\end{aligned}
$$

angenommen. Als korrigierte Werte finde ich die folgenden, die aber durch die nicht bekannten Eigenbewegungen der Vergleichsterne beeinflußt sein können:

$$
\begin{aligned}
& \mu_{\alpha}=+0.35^{12} \pm 0.0004 \\
& \mu_{\delta}=+3.262 \pm 0.006 .
\end{aligned}
$$

Zum Vergleich teile ich die verbesserten Werte mit, die Herr Auwers aus den besten Meridianbeobachtungen seit Bradley hergeleitet hat $^{3}$ ):

Upsala, Sternwarte, I904 Dez. I6.

$$
\begin{aligned}
& \mu_{c}=+0.3492 \\
& \mu_{\delta}=+3.240 .
\end{aligned}
$$

Die verhältnismäßig große Korrektion $z u \mu_{\alpha}$ ist also ziemlich gut verbürgt. In Anbetracht des kurzen Zeitraumes ( 4 Jahre), den meine Beobachtungen umfassen, scheint es mir, daß diese Resultate inbetreff der relativen und absoluten Bewegung von 6 I Cygni ein Zeugnis von der Brauchbarkeit der photographischen Methode für die genauesten astronomischen Messungen darbieten.

Zum Schluß möchte ich die Hauptergebnisse meiner Untersuchungen kurz zusammenfassen. Die Frage nach der Erklärung der beobachteten Schwankungen in dem Abstande $6 I_{1}-6 I_{2}$ Cygni betrachte ich als erledigt und glaube unzweifelhaft nachgewiesen zu haben, daß diese Schwankungen ihre Ursache in dem EinfluB der atmosphärischen Dispersion haben müssen. Betreffs der Parallaxe von 6r Cygni habe ich nachgewiesen, daß die atmosphärische Dispersion (wenig. stens bei den photographischen Bestimmungen) in dem Sinne wirkt, daß, wenn sie nicht berücksichtigt wird, die Parallaxe $\mathrm{zu}$ groß ausfallen muß, und zwar scheint es festgestellt zu sein, daß die Parallaxe kaum größer als o"3 sein kann, was auch durch die letzten Heliometerbeobachtungen (von Herrn Peter $\left.{ }^{4}\right)$ ) bestätigt wird. Jedenfalls scheint es nunmehr nach. gewiesen zu sein, daß, wenn man Beobachtungen, die in verschiedenen Zenitdistanzen angestellt sind, zu Parallaxenbestimmungen verwenden will, es unbedingt notwendig ist, den Stundenwinkelfehler mit in Rechnung zu ziehen.

\begin{tabular}{|c|c|c|c|c|c|c|c|c|c|c|c|c|c|}
\hline \multicolumn{2}{|l|}{1904} & M. Z. Utr. & $\Delta \alpha$ & \multirow{2}{*}{$\begin{array}{c}\Delta \delta \\
+0^{\prime} 53^{\prime \prime} 7\end{array}$} & \multirow{2}{*}{$\frac{\mathrm{Vgl} .}{8.4}$} & \multirow{2}{*}{$\frac{\mathrm{Bb} .}{\mathrm{B}}$} & \multirow{2}{*}{$\frac{\alpha \text { app. }}{20^{\mathrm{h}} 46^{\mathrm{m}} 22^{\mathrm{s}} \text {. I I }}$} & \multirow{2}{*}{$\frac{\log p \cdot \Delta \mid}{9.520}$} & $\delta$ app. & \multirow{2}{*}{$\frac{\log p .4}{0.827}$} & \multicolumn{2}{|c|}{ Red. ad 1. app. } & \multirow[t]{2}{*}{ * } \\
\hline$z$. & 8 & $8^{\mathrm{h}} \quad 3^{\mathrm{m}} 4^{6^{5}}$ & $+1^{\mathrm{m}} 7^{\mathrm{s}} \cdot 75$ & & & & & & $+5^{\circ} 12^{\prime} 29^{\prime \prime} 5$ & & $+15 \cdot 70$ & $+21 " 5$ & \\
\hline & I $\mathbf{I}$ & 8220 & -055.66 & - & 6 & $\mathrm{~N}$ & 203433.91 & $9 \cdot 54^{6}$ & - & - & +1.65 & - & 2 \\
\hline & 13 & 5 I0 33 & -031.80 & +56.7 & I 6.4 & B & $20 \quad 26 \quad 56.14$ & 9.290 & +159 & 830 & $6 \mathrm{I}$ & $+\mathrm{I} 9.4$ & 3 \\
\hline & 13 & $\begin{array}{lll}5 & 29 & 35\end{array}$ & $-\circ \quad 55.79$ & -329.6 & I 2.3 & B & $2026 \quad 52.59$ & $9 \cdot 342$ & $+15^{8} 55.0$ & 0.831 & +1.61 & $+\mathrm{r} 9.4$ & 4 \\
\hline & 20 & $\begin{array}{lll}5 & 47 & 39\end{array}$ & +044.97 & - & I 2 & $\mathbf{N}$ & $1954 \quad 41$ & $9.48 I$ & - & - & +1.5 & - & 5 \\
\hline & 22 & 51223 & -120.31 & 一 & 4 & $\mathbf{N}$ & 194425.09 & 9.459 & - & - & +1.51 & - & 6 \\
\hline & 22 & 52036 & - & $-747 \cdot 4$ & 3 & $\mathbf{N}$ & - & - & $3 \quad 46.3$ & $0.85^{6}$ & - & $+I_{5.5}$ & 6 \\
\hline
\end{tabular}

Meines Erachtens spielt die atmosphärische Dispersion bei den photographischen Fixsternbeobachtungen überhaupt

\begin{tabular}{|c|c|c|c|}
\hline * & $\alpha 1904.0$ & $\delta 1904.0$ & Autorität \\
\hline I & $20^{\mathrm{h}} 45^{\mathrm{m}} \mathrm{x} 2^{\mathrm{s}} .66$ & $+5^{\circ}$ I I $x^{\prime}$ I $4^{\prime \prime} 3$ & $\begin{array}{l}1 / 2 \text { (AG. Alb. } 7289+\text { AG. } \\
\text { Leipzig II 1039I) }\end{array}$ \\
\hline 2 & $2035 \quad 27.92$ & - & AG. Albany 7223 \\
\hline 3 & 202726.33 & + I 5341.0 & 7 I 57 \\
\hline
\end{tabular}
eine wichtige Rolle, die bisher vielleicht im allgemeinen nicht

\begin{tabular}{|c|c|c|c|}
\hline * & $\alpha 1904.0$ & $\delta 1904.0$ & . Autorität \\
\hline 4 & $20^{\mathrm{h}} 27^{\mathrm{m}} 4^{6.5} \cdot 77$ & $+2^{\circ} 2^{\prime} 5^{\prime \prime} \cdot 2$ & AG. Albany 7168 \\
\hline 5 & I9 5354 & - & BD. $-3: 4762$ \\
\hline 6 & I 94543.89 & $-45^{6} \quad \mathrm{I} 4.4$ & $1 / 2\left(M_{1} 21978+\operatorname{Par} .26965\right)$ \\
\hline
\end{tabular}
ihre gebührende Berücksichtigung gefunden hat.

Östen Bergstrand.

1) Mesures micrométriques d'étoiles doubles, Lund i 876, p. $24 \mathrm{I}$.

2) Astr. Nachr. Nr. $\left.2708 .{ }^{3}\right)$ Astr. Nachr. Nr. 3509. $\left.{ }_{4}^{4}\right)$ Astr. Nachr. Nr. 3895.

\title{
Beobachtungen des Enckeschen Kometen (1904 b)
}

am Utrechter Refraktor $(\mathrm{a}=26 \mathrm{~cm}, \mathrm{f}=3 \mathrm{Ig} \mathrm{cm}$ ).

Mittlere Örter der Vergleichsterne. 


\section{Bemerkungen.} gestort.

De2. 11. Komet niedrig. Beobachtung durch Wolken

Dex. 13. Drmmerung. Mond.

Dez. 20. Mond. Kreismikrometer. Beobachtung durch Wolken gestort. Der Stern kann vorlüufig nicht angeschlossen werden.

Utrecht, 1905 Jap. 9.
Dez. 23. Dimmerung. Mond. Kem? Beobachtung durch mangelhafte Fadepbeleuchtung gestort.

Der Komet hatte keinen deutlichen Kern; die Beob. achtungen beziehen sich auf den Brennpunkt des nahezu hyperbolischen Umrisses der ziemlich verwaschenen Nebelmasse. N schitzte im Opernglase die Helligkeit des Kometen am 8. Dezember 7:5; am 22. Dezember etwa 6:5.

\section{Beobachtung des Enckeschen Kometen $1904 \mathrm{~b}$.}

1904 Dez. $96^{\text {h }} 20^{m} 26^{*}$ M. Z. Gottingen $\Delta \alpha=+6: 32 \Delta \delta=-19^{\prime} 44^{*} 38$ Vergleichungen am Heliometer a app. $=20^{h} 42^{m} 51: 69$ d app. $=+4^{\circ} 37^{\prime}$ a5:5 Red. ad L app. + 1:72 +21:6

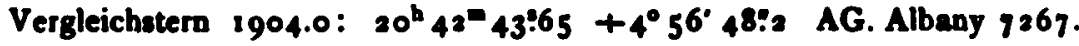

Der Komet ist fir da Heliometer ein sehr schwieriges Objett, da sich fant. gar keine Verdichtung bemerkbar wacht end der ganse Kowet eise verwaschene Lichtunsase ron etwa ' $'$ bis 3 ' Durchmesser darstellt. Nach SSW erschien die Lichtwase etwas weiter ansgedebnt.

Gottingen, Sternwarte, 1904 Dez. 10.

L. Ambrom.

\section{Ephemeride des Planèten (427) [1897 DJ].}

Ausgehend von $M=184^{\circ}$ 14:0 fur 1905 Jan. 14.5 M. Z. Berlin babe ich fur den Planeten (4z7) die folgende Ephemeride fur $12^{\mathrm{b}}$ M. Z. Berlin gerechnet.

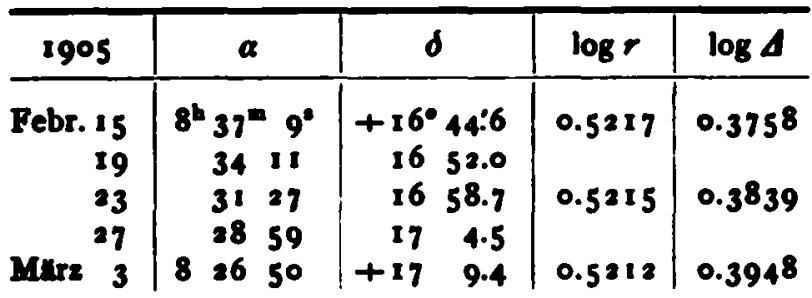

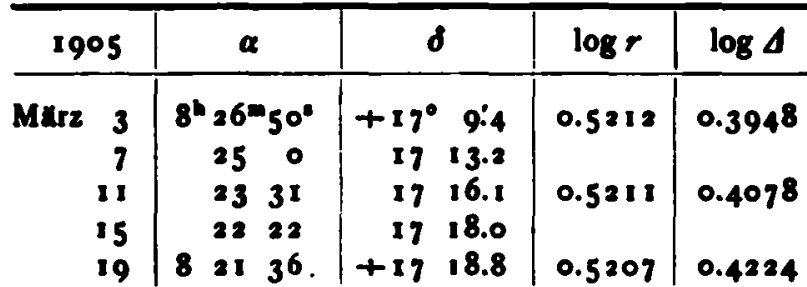

Gr. $13^{m}-14^{m}$. Variation $\pm 1=\mp 6^{\circ}$.

Die Beobachtungen des Planeten 1905 QC werden durch die Ephemeride wie folgt dargestellt (B - R): 1905 Jan. $14+0.3-5^{\circ}$ Jan. $26+0.6-3^{\circ}$ Febr. $9+0.5-2^{\prime}$.

Damit darf die Identitut beider Planeten als erwiesen gelten.

Berlin, Kgl. Recheninstitut, 1905 Febr. 21.

A. Berberich.

\section{Berichtigung za AG. Albany 7195.}

B. prepurant une position de la comete Encke, jai trouve une discordance entre les deux declinaisons suivantes:

$$
\text { - BD. }+294202\left(+2^{\circ} 19: 6\right) \text { et AG. Albany } 7195\left(+2^{\circ} 33^{\circ} 42^{\prime}: 5\right)
$$

concernant la meme etoile de 9.0 ou 8.8 .

Il y a une erreur de $10^{\circ}$ et c'est Albany qui a uoe declinaison trop forte de $10^{\circ} *$ ). Un examea rapide de la region du ciel m'a donac $29^{\prime}$ pour $\Delta \delta$ avec l'etoile voisine BD. +294203 $=$ AG. Albany 7 I94.

Observatoire de Genève, le 12 décembre 1904.

F. Pidoux.

9 Es handelt aich hier offeabar um einen Drackfehler, da die Prizencion in a mit dem richtigen Wert der Deklination, $+2^{\circ} 23^{\circ} 42^{\circ} 5$, Eerechnet its $\boldsymbol{K}$ r.

Inhalt an Nr. 3999. O. Bregenend. Ober die Wirtung der atmouphlyiechen Diapersion anf die Beatimmung der juhrlichen Parallaxen der Fix. sterne. 241. - A. A. Nyjlend, Y. v. d. Bitk. Beobachtangen des Enckeschen Kometen 1904 b. 253. - L. Aminum. Beobachtung des Enckeachen Kometen Igo4 b. 255. - A. Benteriak. Ephemeride des Planeten (427) [1897 DJ]. 255. - F. Pidowr. Berichtigang at AG. Albany 7195. 255. 